Donato Gagliastro

Istituto di studi greco-latini della facoltà di Lettere dell’Università Carolina di Praga

\title{
“Nato nella medesima terra irrigua”. D’Annunzio, ultimo erede di Ovidio
}

A mio padre e mia madre

\section{Premessa ${ }^{1}$}

"Fratello moderno" di Ovidio: così Paratore definì Gabriele d'Annunzio in un saggio ancora attuale [Paratore 1966: 30]. Muovendo da questa definizione, la presente ricerca si propone di svolgere, ricostruendola nelle linee essenziali, la trama di un'ideale fratellanza letteraria. Il testo si sviluppa secondo il progressivo delinearsi di opere e stagioni di d'Annunzio, selezionate ed esemplate attraverso il costante paragone con l'antecedente ovidiano. Il limite di un criterio selettivo non ha impedito, si spera, di estendere comparativamente le indagini ad un itinerario tematico alquanto ampio. Le ricerche si sono giovate di illustri conquiste ermeneuti-

1 A compimento delle ricerche, la mia gratitudine va: a Valerie Bogdan, Annamaria Andreoli, Maria Teresa Imbriani, Vincenzo Paolino, Martin Bažil; alla direzione e al personale della biblioteca centrale di Lettere, Filosofia e Scienze della formazione "Corsano" e della sezione di Italianistica dell'Università degli studi "Aldo Moro" di Bari; ai responsabili e al personale delle biblioteche del Dipartimento di Studi Umanistici - segnatamente, Scienze dell'antichità e Studi classici e - cristiani dell'Università degli Studi “Aldo Moro” di Bari; alla responsabile e al personale della biblioteca comunale "Chiantera" di Polignano a Mare; al personale della biblioteca civica "Rendella" di Monopoli; al mio Nevio. 
che che hanno stimolato un rinnovato confronto tra i testi dei due grandi autori. Nel solco di orientamenti e prospettive inaugurate da insigni maestri e con il conforto delle più recenti acquisizioni, si è tentato di offrire spunti interpretativi atti a evidenziare le chiavi plurime che regolano il rapporto d'Annunzio - Ovidio. Il testo non pretende di esaurire gli sterminati aspetti e problemi insiti sia nella vasta opera dei due autori che nella millenaria distanza che li separa. Tali premesse presuppongono a proprio fondamento un continuo riferimento alla dimensione generale del tema. In questo modo lo studio comparato può ordinare i singoli interventi e coinvolgerli nel quadro complessivo delle relazioni intertestuali che si costituiscono tra d’Annunzio e Ovidio.

\section{Dalla provincia all'Urbe}

In un'analisi comparativa tra d'Annunzio e il poeta augusteo la comunanza dell'origine è un fattore di primario interesse. Come ricorda Elena Ledda, un frammento de Le faville del maglio rivela che, sin dagli anni del collegio Cicognini, l'adolescente Gabriele si sentiva legato da una relazione di "consanguineità" con Ovidio fondata sulla comune origine provinciale, "per essere nato nella medesima terra irrigua" [Ledda 2019: 100]. Come Ovidio, è anche lui Paelignis natus aquosis. La patria peligna è un referente aurorale richiamato da entrambi, non solo nascita ma principio primo e fondamento essenziale: il drammaturgo farà della terra natìa l'ambientazione di acclamate tragedie, segnatamente della Fiaccola sotto il moggio, che rievocano i luoghi peligni, frequentati sin dall'adolescenza e presentati come simbolo di un mondo millenario inalterato "nel passaggio dall'antichità arcaica alla romanità e dalla romanità al medioevo cristiano" [Imbriani 2009: XVII). In Ovidio la memoria radicale della patria peligna risalente ai principi ricorre con insistenza sia negli Amores (in cui è esaltato il carattere rurale e salubre dei luoghi) che nelle dolorose opere del tramonto ${ }^{2}$. Negli anni della formazione al Cicognini di Prato, il brillante collegiale dà prova di una curiosità vorace e di prodigiose capacità assimila- 
trici unite a una precoce propensione per la parola rara e preziosa. È anche un allievo in grado di appassionarsi al severo studio del latino. Legge assiduamente Ovidio ("più di lui precoce" si definisce in rapporto all'augusteo [D’Annunzio 2005a: 1293]), al punto da manifestare alla madre l'intento di cimentarsi nell'impresa di tradurre per intere le Metamorfosi [Andreoli 2000: 25]. Come spesso accadrà negli anni a venire, colmi di preannunci e progetti sistematicamente elusi, anche questa impresa annunciata non sarà mai realizzata. Quel che conta del reboante proclama giovanile è che le letture ovidiane si accordano con l'indole del collegiale che, al debutto con Primo vere all'età di sedici anni, dichiara: "mi sento forte e pieno di ingegno [... ] e lascerò brandelli di carne viva dovunque c'è una gloria da conquistare" [D’Annunzio 1982a: 770].

Quando il diciottenne d'Annunzio lascia la provincia e approda a Roma per iscriversi all'Università, si trova al cospetto di una città che va conoscendo una trasformazione in senso metropolitano. Nel pieno dell'età umbertina Roma vede sorgere, tra gli altri, il quartiere Prati, via Nazionale, via Veneto e, sul fianco del Campidoglio, l'imponente marmo dell'Altare della Patria.

Sulla base di un confronto tra biografie temporalmente divise da lontananze invalicabili3 ${ }^{3}$, il primo ingresso a Roma del provinciale d'Annunzio sembra mostrare un'analogia biografica con Ovidio, venuto giovanissimo a Roma per studiarvi retorica. Oltre le innegabili differenze tra questi due momenti inaugurali, la trasformazione post-unitaria di Roma appare genericamente paragonabile a quella conosciuta dall' "aurea Roma" ovidiana in epoca augustea: l'Urbe che esibiva al mondo conquistato le ricchezze del dominio e fulgeva del Campidoglio, del Palatino e della Curia, destava l'ammirata meraviglia del giovane Ovidio, fiero di essere nato nell'epoca pacificata da Augusto:

Simplicitas rudis ante fuit: nunc aurea Roma est, Et domiti magnas possidet orbis opes. 25-31]. 
Aspice quae nunc sunt Capitolia, quaeque fuerunt:

Alterius dices illa fuisse Iovis.

Curia, concilio quae nunc dignissima tanto,

De stipula Tatio regna tenente fuit.

Quae nunc sub Phoebo ducibusque Palatia fulgent,

Quid nisi araturis pascua bubus erant?

Prisca iuvent alios: ego me nunc denique natum

Gratulor: haec aetas moribus apta meis.

[Ars, III, 113-122]

La ritmica deissi dell'avverbio nunc, ripetuto ad ogni esametro (riecheggia aurea nunc, olim silvestribus horrida dumis di Aen. VIII, 348), enfatizza il giovanile entusiasmo di Ovidio per l'epoca presente e accentua il distacco dal passato sconvolto dalle discordie civili e dalle guerre. Nella Roma che si mostra al giovane d'Annunzio nell'immediata Italia post-unitaria sembra in parte rivivere l'Urbe marmorata di cui si gloriava Augusto nella celebre frase di Svetonio iure sit gloriatus marmoreum se relinquere quam latericium accepisset ${ }^{4}$.

\section{3. "Leggitrici" e doctae puellae}

A Roma, capitale di un'Italia nuova e unita, il giovane d'Annunzio, svogliato studente di provincia, desideroso di compiere una rapida ascesa sociale, abbandona le aule universitarie per frequentare le redazioni giornalistiche. A ventuno anni, dalle colonne della "Tribuna”, compie il suo apprendistato mondano-letterario scrivendo cronache sul grand monde. All'inizio il compito gli riesce insoffribile, giudicato un avvilente spreco del suo ingegno che vorrebbe impiegato per comporre opere letterarie e non per quello che gli appare null'altro che un mansionario impiegatizio. Eppure gli anni a venire dimostreranno che il debutto giornalistico sarà di fondamentale importanza giacché gli assicura un successo mondano prenunzio di quello letterario. Molti testi delle cronache dannunziane confluiranno infatti nel primo romanzo "romano", Il piacere, e, quel che 
è più, attraverso le cronache, egli entra negli splendori dell'alta società romana. Molte dame frivole e salottiere sono affascinate dalla preziosità sensuale del suo eloquio ornato. Con l'acume di un moderno sociologo e la sollecita scaltrezza di un mercante, l'assidua frequentazione di ambienti lussuosi gli fa comprende l'importanza delle mode. È così che il giovane esteta individua nel pubblico femminile il destinatario tipo dei suoi scritti, concepiti come prodotti di consumo per il nascente mercato librario. Egli si propone il compito di appagare quello che definisce, come recita il titolo di un articolo pubblicato su "Il Mattino" nel 1892, il "bisogno del sogno" insito nel vasto pubblico di fruitori del romanzo quale prodotto letterario per il consumo di massa. ${ }^{5}$ Protagonista dai contorni sfumati che emerge da questo pubblico numeroso e anonimo è la donna: una nuova lettrice o "leggitrice" - termine da lui coniato [Andreoli 2000: 111; 2003: XXVI] - che ricerca la letteratura amena per trovarvi emozioni estetiche in grado di innalzare il proprio spirito al di sopra della mediocrità del vivere quotidiano. Il mercato di massa, in quanto tale, neutralizza le differenze di classe poiché il "bisogno del sogno" è un'esigenza comune sia alle nobildonne che alle donne borghesi e plebee. Inseguitore e creatore di mode, d'Annunzio si mostra abilissimo nell'assecondare i gusti del pubblico femminile, ricompensandone le attese con le cronache mondane che, pochi anni dopo, saranno riusate per il suo primo romanzo.

Agli albori della società di massa e prima delle teorie sociologiche dell'industria culturale, l'ambizioso provinciale trasferitosi a Roma smentisce la clamorosa profezia della fine dell'arte nell'epoca del progresso industriale. Egli è tra i primi a intravedere nel genere romanzo la promessa di un clamoroso protagonismo destinato ad aprirgli la via all'ascesa mondana, dimostrando che è possibile invertire "la tendenza del mercato avaro con chi pone in vendita sogni” [Andreoli 2000: 111]. Secondo un insegnamento di Umberto Eco [Eco 2005: 184] "un messaggio non si conclude veramente se non in una ricezione concreta e situazionata che lo

5 In relazione a questi temi, si vedano le acute osservazioni di Annamaria Andreoli nella sua fondamentale biografia dannunziana Il vivere inimitabile [Andreoli 2000: 114, passim]. 
qualifichi." Prima dell'affermarsi delle moderne teorie semiotiche, d'Annunzio intuisce che quando un romanzo, inteso come atto comunicativo, determina un fenomeno sociale, l'interesse deve essere rivolto non solo all'opera in sé ma anche alla generalità dei suoi destinatari.

Precorritore di prodotti culturali contemporanei, egli vedrà anche nella nascente industria del cinema muto non un'invenzione che minaccia di sostituirsi alla letteratura - come temevano i pedanti più indignati - ma una prodigiosa forma d'arte visiva che aveva nel "suo" Ovidio un millenario antecedente: per l'Imaginifico le "cinematografiche" Metamorfosi già preludevano, in germe, a un'estetica del movimento [Andreoli 2000: 502; 2004: 123].

D'Annunzio è dunque un autore consacrato al successo dal nascente pubblico di massa. In rapporto a Ovidio, "nessun poeta latino" - lo ricorda Barchiesi - è così chiaro e fermo nel delineare il progetto di un pubblico orizzontale, arbitro assoluto del successo" [Barchiesi 1994: 35]. Similmente, gli esordi letterari di Ovidio delineano un rapporto privilegiato con il pubblico giovanile connotato anche in senso femminile. A tal proposito, Sharon Lynn James osserva che "elegy overtly identifies its anticipation of women readers" [James 2003:7]. Nell'elegia proemiale del libro II degli Amores, egli si presenta come cantore della poesia amorosa e poeta della sua dissolutezza: Ille ego nequitiae Naso poeta meae ${ }^{6}$. Nel verso spicca nequitia, un termine properziano ${ }^{7}$ che, pur implicando un confronto con Properzio, esclude procedimenti imitativi o parodici; come sostiene Barbara Weiden Boyd in uno studio che recepisce le precedenti intuizioni di Conte, "his efforts in many ways look beyond Propertius' model and offer us instead a vision of elegy that confounds generic boundaries even as it complicates simplistic definitions of imitation." [Boyd 1997: 144].

Abbandonato il proposito di intraprendere un poema epico, Ovidio dichiara di consacrarsi alla poesia amorosa, materia più adatta agli intensi ardori giovanili: me legat in sponsi facie non frigida 
virgo. ${ }^{8}$ L'elegia ovidiana si rivolge esplicitamente a una ragazza, il cui ardore traspare per contrasto dalla litote "non frigida", esortata a leggere i suoi versi. Il termine al singolare invece che al plurale, secondo un procedimento ricorrente nella retorica ovidiana, trasferisce il significato dell'esortazione a un pubblico più esteso di destinatari. Dall'esperienza elegiaca di Ovidio erompe un atteggiamento sprezzante verso la pudicizia tipica di certe convenzioni letterarie a cui molti autori romani soggiacciono quando si tratti di descrivere una ragazza legata, come in questo caso, a un vincolo sentimentale o coniugale. La stessa personificazione dell'Elegia, raffigurata con le fattezze leggiadre di una fanciulla innamorata (forma decens, vestis tenuissima, vultus amantis ${ }^{9}$ ), suggerisce un'identificazione del genere con coloro a cui esso è destinato.

Sia pure con le imprescindibili differenze legate alle rispettive epoche storiche, Ovidio e d'Annunzio si consacrano come autori in grado di cogliere e appagare l'orizzonte d'attesa di nuovi lettori. Quelli ovidiani sono venuti formandosi con l'estendersi dell'educazione ai ceti medi in un'età in cui "i recinti che rinchiudevano la letteratura 'alta' e i loro fruitori non ressero alla spinta impetuosa della diffusione dell'alfabetismo" [Pecere 1996: 5]. Un pubblico colto di giovani lettori e lettrici non più circoscritto alle classi egemoni dell'aristocrazia senatoria, ma emergenti da ceti subalterni aggregati in un più vasto organismo sociale - non vulgus ma media plebs - rinsaldato anche dalla comune propensione alla letteratura d'intrattenimento. Oronzo Pecere precisa che "una parte importante di questo variegato pubblico di lettori è costituito dalle donne (...) Le puellae dettero un impulso determinante alla favorevole accoglienza dei carmina iuvenalia di Ovidio" [Pecere 2018: 379-380]. Il mondo dell'elegia romana contiene una strutturale tensione prodotta dalle relazioni oppositive uomo-donna. In quanto tale, i versi ovidiani esplicitano e incoraggiano la partecipazione del pubblico femminile (Ad mea formonsos vultus adhibete, puellae,

8 Ov. Am. II, 1,5. Rosalba Dimundo svolge puntuali osservazioni sul confronto tra questo verso e il primo distico dell'Ars [Dimundo 2003: 32-33]. 
Carmin $a^{10}$ ) e prefigurano il godimento della lettura dalla prospettiva ideale di doctae puellae, attratte anche dalla dimensione ludica della materia amorosa.

In un'epoca di pace restaurata, il gusto letterario dei nuovi lettori riflette anche la rinnovata concordia civile di una generazione che non ha patito le laceranti esperienze della guerra ed è più incline ad abbandonarsi alla sensualità della giovinezza. Trattati in modo giocoso, gli Amores di Ovidio sono un prodotto dell'estetismo di tradizione ellenistica, come infatti ricorda Wilkinson in un celebre saggio - "the first poem is an introduction to the Amores as a whole, an intimation that in them love will be treated not seriously, as in Propertius and Tibullus, but in a half-humorous, detached, Hellenistic spirit" [Wilkinson 1955: 49 ] $^{\text {"1 }}$ La molteplicità dei motivi deriva dall'intreccio tra gli elementi autobiografici e l'assiduità del poeta-amante con il mondo giovanile dell'Urbe: quas tota quisquam probat Urbe puellas,/ Noster in has omnis ambitiosus amor ${ }^{12}$.

Il mondo elegiaco degli Amores prelude a sviluppi futuri che trovano una prosecuzione nelle Heroides. Se è possibile dedurre i rapporti temporali tra le opere ovidiane da elementi interni al testo, negli stessi anni in cui egli pubblica gli Amores, veniva componendo alcune epistole delle eroine del mito. Amores II, 19 (vv. 21-26) contiene infatti un preannuncio, fondato sul presente scribimus (v. 22), delle epistole di Penelope, Fillide, Enone, Canace, Hipsifile, Arianna, Didone e Saffo.

In un noto verso nel terzo libro dell'Ars, in relazione alle Heroides, Ovidio proclamerà con fierezza la creazione di un nuovo genere: ignotum hoc aliis ille novavit opus ${ }^{13}$. Le sue elegie epistolari ritraggono celebri eroine che abdicano alla loro esistenza nella forma dell'auto-sacrificio, "vivono un'esistenza vicaria, che si nutre di esperienze sostitutive" [Rosati 1989: 44]. Pervase e dominate da un senso

Ov. Am. II, 1, 37-38.

Richiamiamo anche le considerazioni espresse da Barbara Weiden Boyd: "Ovid (...) employs a program rich in Callimachean clichés: a recusatio at the opening of each book; a programmatic statement $(\ldots)$ at or near the close of each book; and programmatic imagery liberally sprinkled elsewhere in the collection, betokening Ovid's aesthetic heritage and allegiance" [Boyd 1997: 135-136].

Ov. Am. II, 4, 47-48.

Ov. Ars, III, 346. 
di offerta sacrificale ai loro amanti, esse sembrano privarsi della loro dignità mitologico-letteraria. Attraverso il lamento elegiaco, Ovidio da un lato trasfigura la grandezza eroica delle protagoniste e dall'altro esibisce le sue pregevoli abilità di autore in grado di esplorare finemente l'animo e la psicologia femminili. "Deciso a privilegiare il rapporto con il pubblico femminile" [Pecere 2018: 380], Ovidio conferisce alle sue Heroides una spiccata e altera teatralità, carattere tipico di alcune figure dannunziane, come si vedrà in seguito ${ }^{14}$.

Per autori quali Ovidio e d'Annunzio, inclini ad appagare il "bisogno del sogno" del pubblico medio e l'evasione dal "grigio diluvio" [D’Annunzio 1988: 34] dell'esistenza comune, questa sovrabbondanza patetica e teatrale diventa una modalità retorica utile ad attrarre e sollecitare l'intervento attivo di entusiaste lettrici. Le Heroides riflettono il mito da una prospettiva femminile che, all'interno di una struttura narrativa di tipo confessionale, sembra poter svelare la soggettività delle eroine e persino l'autonomia delle loro esistenze letterarie in opposizione alle istanze del loro autore biografico. Investite da tale soggettività e autoconsapevolezza, le eroine si configurano come possibili modelli per personaggi letterari di stampo dannunziano ${ }^{15}$. Tra la lettrice antica delle Heroides el'eroina ovidiana si stabilisce un' immaginosa corrispondenza che si potrebbe ricollegare con i meccanismi della cronaca mondana abilmente sfruttati dal giovane d'Annunzio. ${ }^{16}$

Sulla base di precedenti intuizioni, Gianpiero Rosati rileva inoltre la possibile finalità delle Heroides "originariamente concepite come monologhi lirico-drammatici destinati alla rappresentazione scenica" [Rosati 1989: 16].

15 L'accostamento delle Heroides alle eroine dannunziane è ribadito da Gibellini secondo il quale, tuttavia, le prime "superano di gran lunga, per ricchezza d'affetti e altezza d'animo, le moderne dell'invenzione dannunziana” [Gibellini 2019: 66-67]. Recenti studi hanno tentato di interpretare le Heroides come una forma epistolare di espressione femminile, ponendole in una posizione di rilievo nel dibattito contemporaneo sulla scrittura di genere. La lettura di Spentzou [2003], in particolare, è incentrata sulle vite e sulle ambizioni letterarie delle eroine rispetto alle preoccupazioni estetiche di Ovidio.

Come rileva ancora Pecere, le lettrici delle Heroides "partecipano emotivamente alle loro tormentate vicende e si immedesimano nei loro drammi, sublimando le banali vicissitudini delle proprie esperienze amorose" [Pecere 2018: 381 ]. Non sembra inutile richiamare le considerazioni di von Albrecht [1981a] sul modo in cui Ovidio incoraggia l'intesa con i lettori. 


\section{Roma e l'aurea raffinatezza}

Le cronache dannunziane superano gli angusti confini della pagina del giornale e si riversano in gran copia, con sapiente e vantaggioso riuso, in molte parti del Piacere, il primo romanzo scritto nel volgere di pochi mesi (da luglio a dicembre 1888). La gran mole di articoli prodotta nel corso di quattro anni in cui l'esteta ha creduto di sperperare il proprio talento informa l'ambientazione che fa da sfondo allo svolgimento delle vicende di Andrea Sperelli, alter ego di Gabriele, e delle sue amanti.

Il materiale giornalistico innesta nell'azione rutilanti descrizioni di un ambiente sociale romano fatto di arredi sontuosi, ritrovi galanti, squisita urbanità, ricevimenti sfarzosi, uscite a cavallo sull'Aventino e gare ippiche: temi ripresi dalle cronache di costume e di moda scritte da d'Annunzio per le colonne della "Tribuna" con vari pseudonimi (dal celebre "Duca Minimo" al "Marchese di Caulonia”). Nel complesso, tali scene di vita romana esibite al lettore suggeriscono un parallelo tematico con le raffinatezze dei luoghi dell'Urbe, minuziosamente descritti da Ovidio nel primo libro dell'Ars: i portici (Ars I, 67-74), il foro e il marmoreo tempio di Venere (Ars I, 81-88), il teatro (Ars I, 89-134), le corse dei cavalli al Circo Massimo (Ars I, 135-162), i giochi gladiatorii (Ars I, 163-170), lo spettacolo della naumachia (Ars I, 171-176), $\mathrm{i}$ banchetti(Ars I, 229-252). Tutti si offrono al corteggiatore come luoghi favorevoli ai convegni galanti talché "the fine buildings of Rome do not in Ovid's elegy represent the heroic deeds and beneficence of those who built them, but come instead to act as landmarks in the City of Love." [Armstrong 2005: 117]. In questo contesto di emblemi urbani desacralizzati, "anche il cultus, il gusto della raffinatezza [... ] è un piacere adeguato ai tempi, è lo Zeitgeist della nuova, felice "età dell'oro" della Roma di Augusto" [Rosati 2018: 318 ].

Le corse dei carri rivelano legami intertestuali con Amores III, 2 che realizza i precetti teorizzati in Ars I, 135-162. Nell'elegia Ovidio, spettatore della corsa, racconta in prima persona l'assedio galante alla fanciulla seduta accanto a lui. Amores III, 2, 2, cui tamen ipsa faves vincat ut ille precor, e le sequenze ibid. 20-28, 41-42 rinviano 
ai precetti impartiti in Ars I, 146, nec mora, quisquis erit, cuifavet illa, fave e oltre. Le differenze scaturenti dall'analisi comparata devono tener conto delle funzioni di due testi che appartengono a generi letterari diversi. Tali funzioni rispecchiano anche l'evoluzione della personalità dell'autore che passa dal ruolo di poeta-amante che si affina nell'amore come nella composizione della poesia amorosa a quello di distaccato osservatore che, conquistata la preminenza del maestro, impartisce precetti amorosi. Nel Piacere il paesaggio monumentale romano è insistentemente richiamato e descritto (la cupola di San Pietro, la trinità de' Monti, palazzo Barberini, piazza Venezia, il prospetto panoramico del Gianicolo). Tuttavia esso non funge da sfondo ornamentale, al contrario, è strettamente connesso alla psicologia di Sperelli e si accorda con le sue condizioni intellettuali. I luoghi della “Divina Roma” [D’Annunzio 1988: 234] inclusi nel paesaggio hanno una funzione decisiva nei momenti topici della trama. Si veda, ad esempio, l'improvviso incontro inserito attraverso un'analessi - tra Andrea ed Elena avvenuto a via dei Condotti in un mattino intorno a Natale. I due amanti si rivedono a distanza di quasi due anni dall'ultima volta. Pur provando entrambi una "commozione viva" [D’Annunzio 1988: 14], sono costretti a trattenerla perché disdicevole al cerimonioso decoro della strada pubblica. Senza esitare, tuttavia, Elena accetta l'invito di Andrea per il giorno seguente nella sua casa ove "nulla è mutato" [D’Annunzio 1988: 14-15].

Il meccanismo del racconto attribuisce al luogo un valore decisivo - simile a quello che abbiamo visto a proposito dell'Ars - in quanto rende propizio l'incontro tra i due protagonisti e il ridestarsi della loro passione. È un episodio topico e di fatto dà l'avvio alla macchina del racconto che, per oltre metà del romanzo, procede per analessi. Come dimostrato da Annamaria Andreoli [1988: 1146], la scena di via dei Condotti si ricollega chiaramente a un articolo di d'Annunzio scritto per la "Tribuna" il giorno di Natale del 1884 in cui, novello Ovidio memore dei precetti dell' Ars, enuncia i luoghi delle "vie eleganti" di Roma propizie agli incontri amorosi o, come lui scrive, alla "flirtation all'aria aperta" ${ }^{17}$. Sperelli, giovane edoni- 
sta e seduttore raffinato che si appaga delle sensazioni istantanee, è indubbiamente modellato sui contemporanei personaggi dei romanzi d'oltralpe come il des Esseintes di À rebours, riflesso di una cultura, come quella dannunziana, influenzata dalla letteratura francese ${ }^{18}$. Sia pure meno perspicuo, un fondo ovidiano è altresì rinvenibile in Sperelli: in lui si assommano i caratteri del dives amator delineato dalla precettistica amorosa dell'Ars, colui che ripudiando "la rusticitas a vantaggio del nuovo cultus" [Pianezzola 1991: XVIII] sa godere del lusso mondano e della finezza cittadina.

La descrizione che segue, tratta dalle pagine del Piacere, compendia gli attributi che formano il suo potere di acquistarsi "l'animo delle donne". Essi appaiono in sintonia con i comportamenti impartiti con giocosa solennità dal magister amoris ${ }^{19}$ e dettati dall'avamposto del suo carisma: Sperelli è un esperto gaudente che ha cura di sé e ha le qualità dell'inveterato simulatore a cui non ripugna la falsità, addestrato nell'esercizio sapiente di quella che, non a caso, d'Annunzio definisce "arte di amare".

Aprì le labbra per mirare la perfetta lucentezza dei denti e la freschezza delle gengive, ricordando che un tempo ad Elena piaceva in lui sopra tutto la bocca. La sua vanità di giovine viziato ed effeminato non trascurava mai nell'amore alcun effetto di grazia e di forma. Egli sapeva, nell'esercizio dell'amore, trarre dalla sua bellezza il maggior possibile godimento. Questa felice attitudine del corpo e questa acuta ricerca del piacere appunto gli cattivavano l'animo delle donne... La ragione del suo potere stava in questo: che, nell'arte d'amare, egli non aveva ripugnanza ad alcuna finzione, ad alcuna falsità, ad alcuna menzogna. Gran parte della sua forza era nell'ipocrisia [D’Annunzio 1988: 14].

Importa inoltre rilevare un altro esempio, per dimostrare quanto le letture ovidiane che avevano appassionato il collegiale fossero vive nell'immaginazione del romanziere: in apertura del 
romanzo viene introdotto il personaggio di Andrea Sperelli nelle stanze dove egli attende l'arrivo di Elena nella sua dimora a Palazzo Zuccari, in un quartiere molto alla moda nella Roma umbertina. Qui “tutte le cose a torno rivelavano [... ] una special cura d'amore". Nella descrizione degli arredi interni, è citata anche "la piccola tavola del tè $[\ldots]$ con tazze e sottocoppe in maiolica di Castel Durante ornate d'istoriette mitologiche da Luzio Dolci [...] ove sotto le figure erano scritti in carattere corsivo a zàffara nera esametri d'Ovidio" [1988: 5].

In questo quadro iniziale anticipatore del sensuale estetismo del protagonista ogni oggetto è attentamente scelto. È pertanto significativo che il narratore collochi nell'abitazione di Sperelli le preziose maioliche di Castel Durante che saranno offerte all'amante e trascelga quelle che recano una citazione delle Metamorfosi di Ovidio. È da ritenere che lo scrittore, all'interno dell'apparato museografico di Sperelli, non voleva far mancare un riferimento, sia pure marginale, a un autore in sintonia con i suoi propri gusti e quelli del suo personaggio. ${ }^{20}$ Una ripresa puntuale delle stesse maioliche compare più oltre nella scena in cui Maria prepara il tè ad Andrea. L'esattezza descrittiva non tralascia il riferimento già noto al lettore: mentre lei va componendo "l'opera ... le sue mani d'arcangelo si movevano tra le istoriette mitologiche di Luzio Dolci e gli esametri di Ovidio" [1988: 334].

\section{Eroine viventi di là dalla vita}

Tratti di un'eroina ovidiana, Arianna, sembrano riassunti nella figura dolorosa, "disperata e nomade" [D’Annunzio 1989: 292] della Foscarina, celebre attrice che giganteggia nel "romanzo veneziano", Il fuoco. Come Sperelli è la controfigura romanzesca di d'Annunzio,

20 Apprendiamo dalle precise informazioni di Elena Ledda che nel 1888 d'Annunzio "acquista $(. .$.$) due opere ovidiane stampate nel Cinquecento: De arte amandi$ nell'edizione veneziana del 1916 e Le metamorfosi nell'edizione, sempre veneziana, del 1889. Sono tra gli «strumenti di lavoro» presenti sul suo scrittoio durante la stesura del Piacere." [Ledda 2019: 102].

21 La definizione è dello stesso autore all'avvio del Compagno dagli occhi senza cigli [D’Annunzio 2005a: 1451]. 
la Foscarina lo è di Eleonora Duse, la più grande attrice del tempo con cui - fatto notorio - il Vate aveva intrecciato una relazione sentimentale. Riallacciandosi alla tradizione dantesca, d'Annunzio crea una figura in grado di accompagnare e sostenere l'ansia superomistica di Stelio Effrena, il suo slancio verso l'autoaffermazione e la vita eroica. La Foscarina appare mutevole, "creatura notturna e luminosa" [Andreoli 1989: 1200], fragile e languida nel ricordo del passato splendore della bellezza e del corpo giovanile su cui rovina il tempo, estenuata dalla lotta tenace per affermare la perennità dell'arte contro la morte. Si veda questo ritratto modulato da accumulazioni ripetitive che riecheggiano la soggiogante passione distruttiva dell'Arianna di Ovidio:

Un dolore misto di collera travagliò la donna in quella oscurità vibrante e rotta da cui ella non osava escire. Ella soffriva come se fosse riversa sotto un incubo. Le pareva di precipitare al fondo col suo ingombro indistruttibile, con la sua vita vissuta, con i suoi anni di miseria e di trionfo, con il suo volto appassito e con le sue mille maschere, con la sua anima disperata e con le mille anime che avevano abitata la sua spoglia. Quella passione che doveva salvarla, ora la spingeva irreparabilmente verso la ruina e la morte. [D’Annunzio 1989: 343].

In lei la maschera dell'attrice e il volto della donna si confondono e si sovrappongono, la violenta teatralità dei suoi sentimenti elegiaci sembra trovare nell'Arianna ovidiana un antecedente ideale. Il monologo pronunciato dalla Foscarina sul finire del romanzo riecheggia la voce di Arianna, eroina nomade e infelice che vaga solitaria come baccante invasata - aut ego ... erravi ... qualis ... concita Baccha $-{ }^{22}$ il cui dolore è accentuato dalla teatralità dei gesti: fragile e abbandonata, trema come una spiga in balìa dei venti, (corpus ut impulse segetes aquilonibus horret ${ }^{23}$ ) la tunica appesantita dalle lacrime come fossero pioggia (et tunicas lacrimis sicut ab imbre 
graves $^{24}$ ), con le mani lasse di vessare il petto colmo di tristezza e protese al di là del mare per invocare il ritorno delle vele di Teseo (has tibi plangendo lugubria pectora lassas/ infelix tendo trans freta lata manus s. $^{2}$.

Similmente, anche la Foscarina ha conosciuto l'esperienza di un dolore radicale. Nel monologo sono rievocati i momenti significativi della sua vita pubblica e privata, e segnatamente il giorno in cui ha desiderato la morte: in cerca della tomba di Giulietta che aveva interpretato nell'Arena, si sente quasi vinta da un desiderio incoercibile di lanciarsi, insieme alla madre, dal parapetto di un ponte sull'Adige. Tuttavia si ribella al proposito eroico del sacrificio e alla possibilità di una ricompensa per la rinuncia alla vita [D’Annunzio 1989: 455-456]. Si rifiuta - in questo differisce da Arianna - di elevare l'autosacrificio ad adorazione della penitenza, né cerca di spingere il suo amato a rinunciare ai suoi voleri.

Eccettuate le differenze, la Foscarina suscita nel complesso la stessa commozione patetica di Arianna, possiede la stessa capacità di avvolgere le cose con lo sguardo trasferendo su esse la sua stessa essenza. Come per Arianna impietrita (quamquam lapis sedes, tam lapis ipsa $\mathrm{fuit}^{26}$ ), anche per lei il paesaggio intorno acquista un significato tragico. In un'opera come il Fuoco in cui la parola dannunziana ambisce a farsi pittura, esso diventa parte integrante del dolore. Gli oggetti e i relitti che per Arianna simboleggiano la sintesi tra il paesaggio e lo sgomento dell'anima deserta, per la Foscarina rappresentano il franare del tempo sulla vita sospesa sotto un'incombente sentenza di morte. Sono questi fattori dinamici che Jakobson aveva rinvenuto nel "sense of barrenness, the seemingly inevitable doom, the extremity of the situation and the hopelessness" [Jakobson 1974: 227]. L'archetipo mitico di Arianna è ribadito nell'episodio del labirinto in un parco (a Villa Pisani a Strà) in cui Stelio introduce la Foscarina, riluttante ad entrare per tema di perdersi. L'uomo, che ride della sua paura infantile, improvvisamente prende a correre e la abbandona. La donna lo implora 
di tornare indietro, certa che si perderà. Da lontano la voce di Stelio risponde che troverà Arianna. L'angoscia e la gelosia assalgono la donna perché "Arianna" rinvia al nome con cui Stelio ha ribattezzato la cantante Donatella (interprete del lamento di Arianna di Monteverdi [D’Annunzio 1989:422]) da lei stessa presentatogli. "Il terrore che si celava in fondo al suo amore disperato insorse, fu padrone di lei, l'accecò miseramente" [D’Annunzio 1989: 422]. Il percorso labirintico della Foscarina è costruito intorno al mito di Arianna per diventare "vivente allegoria creata dalla sua propria angoscia” [D’Annunzio 1989: 424].

La dimensione eroica della Foscarina-Arianna si converte nel simulacro di un trionfo della vita celebrato all'approssimarsi della fine. Sia d'Annunzio sia Ovidio sembrano risarcire le loro eroine, la loro inesausta volontà di servire e sottomettersi all'amato. Entrambe riempiono del loro eroismo l'una il romanzo l'altra l'epistola. Come per l'Arianna ovidiana, gli elementi della vita della Foscarina concorrono a formare "la figura dell'eroina vivente di là dalla vita" [Andreoli 1989: 469].

In termini generali, se "l'enigma della donna ambigua è la protagonista vera di ogni romanzo di d'Annunzio" [Andreoli 1989: 1399], è forse lecito affermare che nelle donne dannunziane si adombrano alcuni dei tratti costitutivi delle Heroides ovidiane. Le scelte tematiche delle epistole, tanto affini da confondersi e sembrare spesso ripetitive, costituiscono un elemento unificatore intorno al quale si dipana una rete di analogie e rimandi intratestuali. All'interno di questo organismo unitario, nello stesso impatto dannunziano "tra realtà brutale e intimità profonda" individuato dalla Andreoli [Andreoli 1988: 1325], le eroine ovidiane appaiono oscillanti tra abissi di corruzione e innocenza, dissonanti e contraddittorie, gelide e disperate, segrete e voluttuose, attributi che, tutti, variamente presenti, si rinvengono in personaggi quali Elena Muti, Maria Ferres, Ippolita Sanzio, Isabella Inghirami, l'avventuriera della Leda senza cigno. Quest' ultimo titolo, in particolare, adombra un riferimento alla Leda ovidianain Am. I, 3, 23 e II, 4, 41-42.

Il Fuoco compie "il tentativo di sperimentare una sensibilità fonica e acustica davvero nuova, che sa trasformarsi in sostanza intima della parola e della voce nella direzione di una prosa «not- 
turna»" [Andreoli 1989: 1195]. Muovendo da questa conclusione, si può affermare che anche l'intimità della parola e della voce quale si sostanzia nelle Heroides lascia intravedere gli esiti "notturni" della poesia dell'esilio dell'ultimo Ovidio.

\section{II meriggio e l'ombra di Icaro}

Cospicue presenze ovidiane si ritrovano nella grandiosa stagione delle Laudi quando il Vate si riconsegna alla sua più autentica vocazione, non mai abbandonata ma negletta negli anni in cui (almeno fino al Trionfo della morte) il più redditizio genere romanzo è stato preponderante.

Concluso il travaglio compositivo del Fuoco, pubblicato nel 1900 dopo molti rimaneggiamenti, la poesia di d'Annunzio si schiude strabocchevole nella prodigiosa estate del 1902 quando nascono con mirabile rapidità le migliaia di versi di Alcyone, il terzo libro delle Laudi. Nelle lettere private al suo traduttore Hérelle e all'editore Treves, la febbrile copiosità della poesia alcionia è celebrata con la metafora del "largo fiume" [1984: 1145].

Nel monologo delle Laudi dove "innologica, elencatoria, ad alta voce, la pronuncia dell'io risuonerà in una cornice diaristica” [Andreoli 1988: 1294], il canto lirico riflette uno stato di grazia edenica, conquistato nel tempo trasognato di una vacanza estiva, che prende forma intorno al "protagonismo metamorfico" [Andreoli 1989: 1150] di un io che si autocelebra nell'accordo panico e mimetico con la natura. Intorno ad essa si diffondono figurazioni favolose nell'estasi dei sensuali meriggi estivi. Gli eventi metamorfici del d'Annunzio alcionio che si trasfonde in Glauco, simbolo dell'aspirazione sublime a divinizzare la vita umana ${ }^{27}$, poggiano su sensazioni fisiche che attingono a un fondo lessicale classico di matrice primariamente ovidiana che infittisce la trama analogica del testo

27 Belponer sottolinea che "nel creare il nuovo Glauco, d'Annunzio concentra l'attenzione sull'esperienza del «trasumanar», attraverso il filtro della memoria dantesca, ma aspira proprio a «significar per verba» quell'esperienza che Dante dichiara impossibile, in una sorta di emulazione competitiva" [Belponer 2019: 77]. 
poetico ${ }^{28}$. Il lirismo di molte laudi si prolunga fino all'estremo limite attraverso le incalzanti enumerazioni che alimentano l'inesauribile volontà di nominare le cose e in questo procedimento il vocabolario alcionio si giova di fonti classiche che si intrecciano.

Gli sfrenati ditirambi fanno un intenso uso di rielaborazioni e riecheggiamenti delle Metamorfosi. ${ }^{29}$ In generale i 4 ditirambi sono preannunciati da una lirica introduttiva con un titolo latino, in tre casi è Ovidio a prestare il titolo: "Terra vale", (il saluto di Glauco alla terra prima di inabissarsi ${ }^{30}$ ) "Stabat nuda aestas" e Altius egit iter. È da notare che aestus - etimologicamente apparentato ad $a_{e s t a s^{31}}$ è un termine ricorrente nel lessico torrido degli Amores ${ }^{32}$ e parole dannunziane da esso derivate, quali "estuare" ed "estuoso", si riversano nel lessico meridiano di Alcyone 3 $^{33}$.

L'apice vertiginosa della rielaborazione mitologica è il "ditirambo di Icaro" dove d'Annunzio, lo dichiara nelle Faville del maglio, sperimenta "lo struggimento di bevere il soffio dell'altezza titanica" [d'Annunzio 2005b: 1227]. All'estremo della sua ansia superomistica, il Vate anela ad Icaro, rappresentante del suo desiderio di oltranza, "sentendosi fratello del volatore antico per «la comune d'altezze e d'abissi avidità»" [Gibellini 2019: 70]. Nel 1922 il reduce di Fiume commemorerà in latino un'infausta caduta da una finestra dell'eremo di Gardone. Con termini ovidiani, poi incisi nell'ingresso della Prioria, si autocelebra "volucer dimissus ab alto", volatore precipitato come Icaro e come l'Arcangelo del suo nome. ${ }^{34}$

Il ragguardevole commento di Giulia Belletti, Sara Campardo e Enrica Gambin nella più recente edizione critica di Alcyone a cura di Pietro Gibellini (2017) dimostra che Ovidio primeggia sugli autori classici per numero di riferimenti.

Non prospettiamo i numerosi esempi tratti dalle Metamorfosi che riecheggiano in Alcyone e su cui si sono esercitati illustri commentatori. Per i riscontri testuali si rimanda ai commenti di Roncoroni [1982], Caliaro [1995], Belponer [1995] e le già citate Belletti, Campardo e Gambin [2017]. Per una ricostruzione aggiornata dei più recenti contributi alla filologia dannunziana si veda Maiolini [2019]. Ov. Met. viII, 948.

Si veda il duplice lemma "Aestās, -ātis; aestus, -ūs" nel Dictionnaire étymologique de la langue latine Ernout-Meillet.

32 Am. I, 5, 1, II, 11, 40 (alcuni codici leggono eurus invece di aestus), III, 2, 39, III 5, 7-8, 36 .

33 Registriamo le occorrenze in "Stabat nuda aestas", v. 3, "Bocca di serchio", v. 140, "Ditirambo II", v. 11.

34 Si veda la descrizione tratteggiata da Andreoli [2004: 164]. 
I luoghi incantati di Alcyone riappaiono anche nelle pagine di Forse che sì, forse che no, romanzo del 1910, che pone il culto estetico della velocità e dell'ebbrezza del volo con gli aeromobili al centro di una nuova ideologia della modernità celebrando il "Nuovo Dedalo" nella figura di Paolo Tarsis. I luoghi alcioni sono catturati dalla prospettiva aerea e le loro descrizioni non sfuggono all'accrescimento cumulativo tipico del canzoniere [D’Annunzio 1989: 553, 673-674, 865].

L'eccezionalità di Alcyone ha determinato - leggendo la Andreoli - "una propensione autocelebrativa senza precedenti nella storia dannunziana" [Andreoli 1984: 1166]. La voluttà dell'abbandono contemplativo di Alcyione e il desiderio di ritornare alla stagione incantata delle Laudi riecheggiano nelle pagine degli scritti memoriali in cui il Vate in ombra che si attarda nella solitudine torna alla sua immagine perduta per rievocare, rimpiante, le opere giovanili e le irripetibili laudi [D’Annunzio 1989: 1027].

\section{Verso il tramonto}

Alcune cospicue convergenze possono infine scaturire dal confronto tra i due autori nella loro ultima stagione. Per entrambi gli anni che precedono la morte trascorrono all'insegna dell'esplorazione notturna e di una suprema singolarità.

Nelle opere del tramonto, sia Ovidio sia d'Annunzio inclinano verso l'ombra. Lontani da Roma senza più farvi ritorno, entrambi vivono isolati e reclusi, l'uno a Tomi, relegato da Augusto ai margini estremi dell'Impero, l'altro, il reduce di Fiume, in volontario esilio sulle remote rive del Garda, "un Mediterraneo in miniatura" [Gibellini 2019: 66].

Nell'esilio in cui le loro vite declinano, Ovidio conserva un rapporto tenace con la poesia, mentre d'Annunzio di fatto se ne congeda. Elegie della solitudine, i Tristia e le Epistulae ex Ponto risuonano del lamento di una voce bandita che invoca il conforto del passato per resistere alla consunzione del presente. ${ }^{35}$ Paolo Fedeli ricorda il giudizio di d'Annunzio sui Tristia levatosi in con- 
trasto con la critica ufficiale in una temperie in cui la produzione ovidiana dell'esilio era stata "troppo a lungo negletta o troppo rapidamente liquidata perché ritenuta indegna di appartenere alla letteratura ispirata e impegnata, in quanto esercizio sbiadito di doti un tempo somme" [Fedeli 1999: CXv]. Nelle Faville il Vate dichiara di prediligere i Tristia, "attratto dal "crudo esilio tomitano e dall'angoscia del voluttuoso cuore irto di ricordanze sotto quel gran vento di Scizia" [D’Annunzio 1924 cit. in Fedeli 1999: CXVI].

Lasciato incompiuto il progetto delle Laudi mentre dei nuovi romanzi non restano che gli annunci, il recluso di Gardone si consacra alla scrittura memoriale e dà vita alle "prose di ricerca" sperimentando una sorta di "metaletteratura" [Andreoli 2005a: XXXII] fatta di introspezioni e straniamenti. Tra inquiete presenze e oggetti estranei, d'Annunzio e Ovidio contemplano il ritmo interiore dell'esistere e identificano la loro arte con la lontananza del passato richiamato per risarcirli dei torti del presente. La solitudine di due autori che propendevano all'acclamazione del pubblico scopre un'interiorità che riflette su se stessa e prepara la strada all'epilogo. In più, con la memoria dei propri illustri trascorsi, i due poeti esuli sembrano configurare, da prospettive diverse, una comune epica autocelebrativa.

"Gettato lontano sulle sponde sarmatiche" (sic ego, Sarmaticas longe proiectus in oras $^{36}$ ), lo sguardo tormentato di Ovidio si affissa su Roma e sul tempo rimpianto dei successi e della giovinezza. Trasfigurate dall'esilio, le elegie della lontananza oppongono una resistenza all'urto con i luoghi ostili e con la lacerazione che attanaglia la sua vita repulsa ai margini. Chiuso nei confini insuperabili dell'esilio, Ovidio non può fare a meno di guardare con spregio, raffigurandoli come ostili e barbari, i luoghi battuti dal "gran vento di Scizia”, già nominati - involontario presagio - in due versi degli Amores in contrapposizione alla salubrità dei paterni campi ${ }^{37}$. L'immagine di Roma, patria perduta, persiste nell'assenza perpetua. La poesia diventa espressione di un dolore abissale che rispecchia la costrizione dell'uomo chiuso nel corpo e nello spirito entro la prigionia di luoghi estranei. L'unico monologo possibile a Ovidio 
è quello del lamento ove la voce dell'io risuona per affidare alle elegie il compito supremo di preannunciare il suo "morire lontano"38 In un estremo slancio consolatorio, i Tristia provano ad innalzarsi al di sopra della miseria della realtà ma non ne possono eliminare la costitutiva tristezza.

D’Annunzio non ha che la propria magnificenza da opporre alle vicende politiche che lo hanno emarginato. Dopo la marcia su Roma, è dissuaso dall'azione politica. Dall'esilio volontario si consegna alla prosa memoriale inaugurando un nuovo autobiografismo per elevare un monumento di parole a se stesso. In cambio della sua uscita dall'agone politico, riceve l'onore dell'edizione nazionale delle sue opere a cui lui stesso sovrintende (gli Indici usciranno nel 1936). Parallelamente, vede ampliarsi la villa sul Garda che grazie ai lauti fondi elargiti dal governo - acquista le dimensioni di una cittadella monumentale e diventa il Vittoriale, variante del Vittoriano, innalzato al Re dell'Unità d'Italia, Vittorio Emanuele II.

Il ripiegamento memoriale sostituisce la narrativa in prima persona con la prosa diaristica che erompe inarrestabile. Il nuovo corso della scrittura dannunziana attraversa tanti scritti stilisticamente affini in cui il tempo si sublima in un diario ininterrotto a partire dalle Faville del maglio, uscite sul "Corriere della Sera" tra il 1911 e il 1914: ${ }^{39}$ sono questi gli scritti che, secondo un diffuso luogo critico, segnano la nascita della stagione "notturna" di d'Annunzio che, contrapposta ai meriggi alcioni, si perpetua fino alla morte. Non diversamente, l'infelicità ovidiana dell'esilio contrappone i Tristia e le Epistulae alla letizia delle poesie amorose attraverso due opere che oltretutto riconquistano il genere elegiaco alla caratteristica originaria del lamento e del compianto.

Echi di lontananze ovidiane si possono rinvenire in frammenti dell'opera paradigmatica dell'ultima stagione del Vate, il Notturno, prodotto, in parte, della cecità sacrificale del mutilato. ${ }^{40}$ Nella e del Notturno che recano la data dell'avvenimento passato rievocato nel tempo presente.

40 È noto che il Notturno è stato in parte composto durante la cecità conseguente alla ferita al capo per un incidente aereo occorso nel gennaio 1916. Relegato al buio 
"seconda offerta", d'Annunzio rievoca la tragica fine di amici aviatori che cadono sul campo di Gonars. Il sacrificio assimila i patrioti all'Icaro ovidiano tanto caro al poeta-milite [D'Annunzio 2005a: 215-217] che aveva salutato nella nascita dell'aviazione una forma di divinizzazione del moderno uomo-icaro. ${ }^{41}$ Il poeta-milite, inizialmente destinato a partecipare all'impresa, si vede strappato all'incontro con la morte: "sono un'ombra alata che ascolta il suo mito" [217]. L'episodio è seguito dalla rievocazione - cronologicamente anteriore - dell' imperiosa orazione dannunziana che incitava all'intervento dell' Italia in guerra, pronunciata dal Campidoglio nel «Maggio radioso» del 1915 al cospetto della folla esultante..$^{42}$ Concluso il ricordo del giorno trionfale davanti alla moltitudine, il prosatore notturno indulge alla descrizione della solitudine che ne era seguita quella stessa notte. Dall'oscura profondità del presente scaturisce lo struggente ricordo del momento in cui d'Annunzio ritrova Roma, che sentiva perduta durante gli anni dell'esilio francese. Dopo l'ebbrezza del giorno si incammina verso l'Aventino, "solo come un solitario amante". Il racconto, intessuto di lirismo ("ricercando me stesso, non ritrovavo se non la mia malinconia” [223]), offre paragoni con la solitudine di Sperelli, amante abbandonato, sul finire del Piacere [D’Annunzio 1988: 258] nonché con l'apostrofe che d'Annunzio rivolse all'“Anima trista" del suo alter ego nelle terzine Ad Andrea Sperelli il cui "cor franto/ geme il verso che esalta e che consola” [D’Annunzio 1982: 588]. La città tumultuosa che in quel giorno di maggio aveva acclamato l'oratore infine si acqueta e al suo posto gli appare la città degli intensi amori giovanili, che fu perpetua di voluttà: "mi teneva un

e all'immobilità, d'Annunzio compone per frammenti il "commentario delle tenebre", scrivendo su liste di carta strette tra il pollice e il medio.

41 Si veda l'intervista al «Matin» del 3 maggio 1910, ora in Scritti giornalistici 18891938 [D'Annunzio 2003: 1439]. Giova inoltre ricordare che, agli albori dell'industria aviatoria, d'Annunzio trae da Ovidio il fortunato vocabolo "velivolo", pseudo-neologismo reimmesso nell'uso e proposto in alternativa ad "aeroplano", di più ardua pronuncia [Andreoli 2005: 151].

42 Va osservato che nell'oratoria dannunziana inneggiante alla guerra non è infrequente il riferimento al mito ovidiano di Meleagro (Met. viII, 445-546), ripreso poco oltre (p. 225), anche nel Notturno. Per il testo integrale dell'orazione si veda Dalla ringhiera del Campidoglio il XVII maggio MCMXV in Per la più grande Italia, ora in Prose di Ricerca [2005a: 51-54]. 
amore sensuale di Roma, un amore voluttuoso della mia Roma, simile a quello che consumò le forze della mia giovinezza" [223]. Non solo l'Urbe del Piacere ma, a ben vedere, anche la città che era stata la musa ispiratrice della Chimera - "d'improvviso tutta quanta Roma,/ al lume di quel gran desire, già / ridemi” scriveva nell'ode Donna Francesca [D’Annunzio 1982: 476 vv. 29-31] - e delle Elegie romane in epigrafe alle quali il giovane d'Annunzio aveva apposto un verso ovidiano dell'esilio: "Quid melius Roma?”.43 Come in alcune elegie dei Tristia, ${ }^{44}$ anche questo frammento dannunziano, dunque, configura Roma e la giovinezza come due temi fondanti che procedono in parallelo. Entrambi rinviano a un luogo e a un tempo del passato separati da quelli del presente, simboli l'uno e l'altro di una distanza incolmabile e di una condizione non più posseduta e rimpianta.

Lo struggimento dei due spiriti esuli che si corrispondono anche sul declinare della vita è ribadito infine da Gibellini: "Anche l'umor malinconico dei Tristia, ideale complemento e rovescio della gioiosa sensualità degli Amores, trova rispondenza nel d'Annunzio della Contemplazione della morte, del Notturno e di molte lettere senili intrise di spleen, che possono a loro volta equipararsi alle Epistulae ex Ponto" [Gibellini 2019: 66-67]. È un sigillo finale per entrambi quello impresso nella compagine dei ricordi e dei frammenti registrati nelle opere estreme che - con le parole profetiche di un cupo d'Annunzio nel 1922 - lasciano in loro quel che c'è di più "lontano e di misterioso e di inafferrabile" [D'Annunzio 1922 cit. in Andreoli 2005b: 3461].

\section{Bibliografia ${ }^{45^{*}}$}

Andreoli Annamaria (2000), Il Vivere Inimitabile. Vita di Gabriele d'Annunzio, Mondadori, Milano.

Andreoli Annamaria (2004), D’Annunzio, Il Mulino, Bologna.

45 "Salvo diversa indicazione, tutte le citazioni di d'Annunzio presenti nel testo si riferiscono all'edizione delle opere di Gabriele d'Annunzio pubblicate nei "Meridiani Mondadori”. 
Andreoli Annamaria, Lorenzini Niva (1982) (a cura di), Gabriele d'Annunzio. Versi d'amore e di gloria, vol. 1, Mondadori, Milano.

Andreoli Annamaria, Lorenzini Niva (1984) (a cura di), Gabriele d'Annunzio. Versi d'amore e di gloria, vol. 2, Mondadori, Milano.

Andreoli Annamaria, Lorenzini Niva (1988) (a cura di), Gabriele d'Annunzio. Prose di romanzi, vol. 1, Mondadori, Milano.

Andreoli Annamaria, Lorenzini Niva (1989) (a cura di), Gabriele d'Annunzio. Prose di romanzi, vol. 2, Mondadori, Milano.

Andreoli Annamaria (2003) (a cura di), Gabriele d'Annunzio. Scritti giornalistici 1889-1938, vol. 2, Mondadori, Milano.

Andreoli Annamaria, Zanetti Giorgio (2005) (a cura di), Gabriele d'Annunzio. Prose di ricerca, vol. 1, Mondadori, Milano.

Andreoli Annamaria, Zanetti Giorgio (2005) (a cura di), Gabriele d'Annunzio. Prose di ricerca, vol. 2, Mondadori, Milano.

Armstrong Rebecca (2005), Ovid and his love poetry, Bloomsbury, London.

Barchiesi Alessando (1994), Il Poeta e il Principe. Ovidio e il discorso augusteo, Laterza, Bari.

Belponer Maria (2019), L'eredità di Ovidio in Giovanni Pascoli e Gabriele d'Annunzio, "Archivio d'Annunzio", vol. 6, pp. 67-84.

Boyd Barbara Weiden (1997), Ovid's literary loves. Influence and innovation in the Amores, The University of Michigan Press, Ann Arbor.

Dimundo Rosalba (2003), Ovidio. Lezioni d'amore. Saggio di Commento al I libro dell'Ars Amatoria, Edipuglia, Bari.

Eco Umberto (2005, I ed. 1976), Il superuomo di massa. Retorica e ideologia nel romanzo popolare, Bompiani, Milano.

Fedeli Paolo (1999) (a cura di), Ovidio. Opere. I. Dalla poesia d'amore alla poesia dell'esilio, Einaudi, Torino.

Gibellini Pietro, Belponer Maria (1995) (a cura di), Gabriele D’Annunzio. Alcyone, Garzanti, Milano.

Gibellini Pietro (2017) (a cura di), Gabriele D’Annunzio. Alcyone, commento di Giulia Belletti, Sara Campardo, Enrica Gambin, Marsilio, Venezia.

Gibellini Pietro (2019), D’Annunzio, moderno Ovidio, in: Da Ovidio a d'Annunzio. Miti di metamorfosi e metamorfosi dei miti. Atti del convegnodi studi, Silvana Editoriale, Cinisello Balsamo, pp. 65-71.

Imbriani Maria Teresa (2009) (a cura di), Gabriele d'Annunzio, La fiaccola sotto il moggio, Il Vittoriale degli Italiani, Verona.

James Sharon Lynn (2003), Learned girls and male persuasion: gender and reading in Roman Love Elegy, University of California Press, Berkeley and Los Angeles. 
Ledda Elena (2019), Ovidio nella biblioteca del Vittoriale, in: Da Ovidio a d'Annunzio. Miti di metamorfosi e metamorfosi dei miti. Atti del convegno di studi, Silvana Editoriale, Cinisello Balsamo, pp. 99-112.

Maiolini Elena (2019), Stato, problemi, applicazioni critiche della filologia dannunziana, "Archivio d’Annunzio", vol. 6, pp. 101-128.

Paratore Ettore (1966), Studi dannunziani, Morano, Napoli.

Pecere Oronzo, Stramaglia Antonio (1996) (a cura di), La letteratura di consumo nel mondo greco-latino. Atti del convegno internazionale, Università degli Studi di Cassino, Cassino.

Pecere Oronzo (2018), Libro e lettura nella poesia di Ovidio, in: Ovidio 2017. Prospettive per il terzo millennio. Atti del convegno internazionale, a cura di Paolo Fedeli, Gianpiero Rosati, Ricerche\&Redazioni, Teramo, pp. 375-403.

Pianezzola Emilio (1991) (a cura di), Ovidio. L'arte di Amare, VallaMondadori, Milano.

Rosati Gianpiero (1989) (a cura di), Ovidio. Lettere di eroine, Rizzoli, Milano.

Rosati Gianpiero (2018), Ovidio e l'invenzione del corpo femminile, in: Ovidio 2017. Prospettive per il terzo millennio. Atti del convegno internazionale, a cura di Paolo Fedeli, Gianpiero Rosati, Ricerche\&Redazioni, Teramo, pp. 313-331.

Spentzou Efrossini (2003), Readers and Writers in Ovid's Heroides.

Transgression of Genre and Gender, Oxford University Press, Oxford.

Von Albrecht Michael (1981), Ovide et ses lecteurs, "Revue des études latines", n. 59, pp. 207-215.

Wilkinson, Lancelot P. (1955), Ovid recalled, Cambridge University Press, Cambridge.

Donato Gagliastro

"Nato nella medesima terra irrigua”. Gabriele D’Annunzio, the Last Heir of Ovid

Throughout Gabriele d'Annunzio's vast and multiform productions, Ovid's presence shines through with the strength of a model both ancient and modern. Since his years at the "Cicognini", he feels bound to Ovid by a similarity "in lyrical ways" and by a relationship of "consanguinity" based on their common origins. The assiduity in Ovid's readings, testified to by the large number of volumes of the Augustan poet in the library of the Vittoriale, is reflected in a dense network of echoes - some perspicuous, others subtle - that can be found in the Vate's most famous works. During his twilight years, d'Annunzio's nocturnal exploration finds singular affinities 
with the Ovidian elegies of exile to which he feels attracted the most. Even in the nascent silent film industry, d'Annunzio, creator and forerunner of fashions, sees a prodigious form of visual art that has in Ovid a millenary antecedent. Beyond the unavoidable differences separating the two temporally distant authors, the paper attempts to outline an overall profile of the correspondences and possible equations between d'Annunzio and Ovid without pretense of exhaustiveness. In the wake of perspectives inaugurated by distinguished masters, it is an attempt to offer cues for interpreting, from the vestiges of classicism, d'Annunzio's art within the Ovidian framework.

Keywords: Rome; readership; elegy; nocturnal poetry; memory.

Donato Gagliastro - Dottorando presso l'Istituto di studi greco-latini della facoltà di Lettere dell'Università Carolina di Praga, docente di ruolo di italiano e latino nei licei italiani. Privilegiando la prospettiva fondata sul metodo comparativo, attualmente si sta occupando della ricezione dei classici, segnatamente di Ovidio, in un manoscritto latino di epoca francescana nonché del francescanesimo nell'estetismo dannunziano. Giurato in un certamen ovidianum e autore di numerosi articoli di critica letteraria, recentemente ha tenuto un corso sulle Heroides e pubblicato un articolo sui Tristia, The last farewell from the exile: resistance and twilight in Ovid's heroic poetry (2019). Contatto: gagliasd@ff.cuni.cz. 